\title{
On the decay rate of Hankel singular values and related issues *
}

\author{
A.C. Antoulas \\ Electrical and Computer Engineering \\ e-mail: aca@rice.edu \\ http://www-ece.rice.edu/ aca
}

\author{
D.C. Sorensen and Y. Zhou \\ Computational and Applied Mathematics \\ e-mail: \{sorensen,ykzhou\}@rice.edu \\ http://www. caam.rice.edu/ ${ }^{\text {sorensen }}$
}

\author{
Rice University \\ Houston, Texas, 77005
}

May 13, 2001

\begin{abstract}
This paper investigates the decay rate of the Hankel singular values of linear dynamical systems. This issue is of considerable interest in model reduction by means of balanced truncation, for instance, since the sum of the neglected singular values provides an upper bound for an appropriate norm of the approximation error. The decay rate involves a new set of invariants associated with a linear system, which are obtained by evaluating a modified transfer function at the poles of the system. These considerations are equivalent to studying the decay rate of the eigenvalues of the product of the solutions of two Lyapunov equations. The related problem of determining the decay rate of the eigenvalues of the solution to one Lyapunov equation will also be addressed. Very often these eigenvalues like the Hankel singular values, are decaying rapidly. This fact has motivated the development of several algorithms for computing low rank approximate solutions to Lyapunov equations. However, until now, conditions assuring rapid decay have not been well understood. Such conditions are derived here by relating the solution to a numerically low rank Cauchy matrix determined by the poles of the system. Bounds explaining rapid decay rates are obtained under some mild conditions.
\end{abstract}

\section{Contents}

1 Introduction 2

2 Cauchy Matrices $\quad 3$

3 Eigenvalue decay rates for system grammians 4

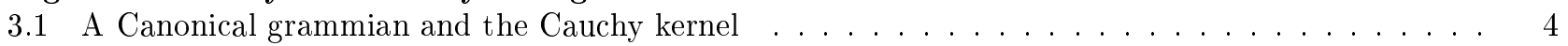

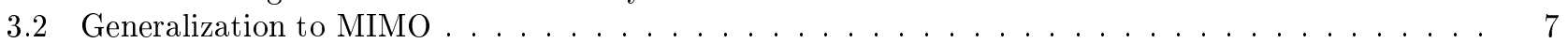

4 Decay rate of the Hankel singular values $\quad 8$

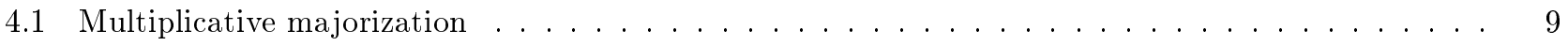

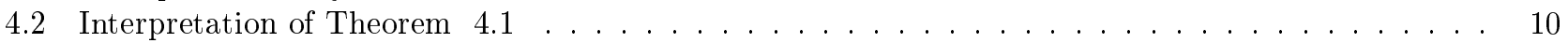

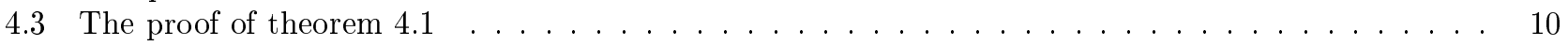

${ }^{*}$ This work was supported in part by the NSF through Grants DMS-9972591 and CCR-9988393. 


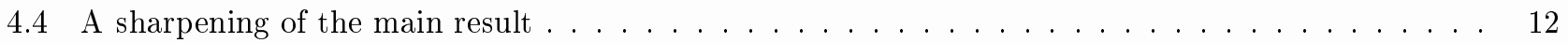

4.5 Connection with Fuhrmann's results $\ldots \ldots \ldots \ldots \ldots \ldots \ldots$

5 Numerical Examples and Discussion $\quad 13$

5.1 Cases of non-decay or slow-decay . . . . . . . . . . . . . . . . . . . . 18

6 Conclusions $r$

7 References $\quad 20$

\section{Introduction}

Linear time invariant (LTI) systems

$$
\boldsymbol{\Sigma}:\left\{\begin{array}{l}
\dot{x}(t)=A x(t)+B u(t) \\
y(t)=C x(t)+D_{0} u(t)
\end{array} \Leftrightarrow \mathbf{\Sigma}:=\left(\begin{array}{c|c}
A & B \\
\hline C & D_{0}
\end{array}\right)\right.
$$

where $A \in \mathbb{R}^{n \times n}, B \in \mathbb{R}^{n \times m}, C \in \mathbb{R}^{p \times n}, D_{0} \in \mathbb{R}^{p \times m}$, arise frequently in many branches of engineering. Closely related to this system are two continuous-time Lyapunov equations:

$$
A P+P A^{T}+B B^{T}=0, \quad A^{T} Q+Q A+C^{T} C=0
$$

Under the assumption that $A$ is stable, it is well known that the above equations have unique symmetric non-negative definite solutions $P, Q \in \mathbb{R}^{n \times n}$, called the controllability and observability grammians, respectively. In many applications, such as circuit simulation, or time dependent PDE control problems, $n$ is quite large, while the number of inputs $m$ and outputs $p$ usually satisfy $m, p \ll n$.

We begin by considering only one of the two equations in (1):

$$
A P+P A^{T}+B B^{T}=0
$$

In general and especially when $n$ is large it is unwise to solve for $P$ directly since this requires $O\left(n^{3}\right)$ flops and $O\left(n^{2}\right)$ storage. Many have observed that the eigenvalues of $P$ generally decay very fast [10], [18]. Because of this, $P$ may be approximated by a low rank matrix. Several iterative methods for computing a low rank approximation to $P$ have been proposed [10], [12], [14], [18], [19]. See [3] for a recent survey of such methods. There are some results on the eigenvalue bounds for the solution of Lyapunov equations [7], [15], but these do not explain why (2) permits the very low rank approximate solutions observed in practice. The eigenvalue bounds surveyed in [15] focus mainly on $A P+P A^{T}+S=0$ with $S \in \mathbb{R}^{n \times n}$ positive semidefinite, and the special low rank structure of $S=B B^{T}$ is not fully exploited. Moreover the lower bounds for small eigenvalues of $P$ are trivially zeros. Penzl [17] took into account the low rank structure of $B B^{T}$. He established upper bounds on the ratios $\frac{\lambda_{k}(P)}{\lambda_{1}(P)}$ for symmetric $A$, but this approach depends heavily on symmetry and is not easily generalized to the non-symmetric case.

The first goal of this paper, is to derive decay rates that are direct estimates of the error of the best rank $k$ approximation to $P$. In contrast to the the Penzl estimates, our results do not establish explicit bounds for the eigenvalues of $P$. Instead, we obtain an outer product representation of the solution of the form

$$
P=\sum_{j=1}^{n} \delta_{j} z_{j} z_{j}^{*} \text { with } \delta_{1} \geq \delta_{2} \geq \ldots \geq \delta_{n}>0 .
$$


When $A$ has an eigenvector basis that is not too ill-conditioned, the norms of vectors $z_{j}$ are uniformly bounded by a modest constant and hence the ratio $\delta_{k+1} / \delta_{1}$ gives an order of magnitude relative error estimate for a rank $k$ approximation to $P$.

These results lead directly to an explanation of why it is often possible to approximate $P$ with a very low rank matrix. They are closely related to the eigenvalue decay rates of Penzl [17] which are the only such results we are aware of. His results are stated in terms of the condition number of $A$ which is assumed symmetric. Our bounds are functions of the eigenvalues of $A$ and make no symmetry assumptions. We give some numerical results and compare the two. These results show that the bounds given here seem to give a significantly better indication of the actual decay rate of the eigenvalues of $P$.

Next we turn our attention to the Hankel singular values which are defined as the square roots of the eigenvalues of the product of grammians $P Q$ in (1). Due to the importance of the Hankel singular values in model reduction, in particular balanced model reduction of large-scale systems, there has been some activity recently on the issue of the decay rate of these singular values. It has been observed that in many cases these quantities decay very fast and therefore the corresponding systems are easy to approximate. Two recent approaches are [4] and and [5].

Our second goal in this paper is to present a different approach to the problem of determining the decay rate of the Hankel singular values. It is based on a new set of system invariants. If the transfer function of the system in question is $Z=\frac{p}{q}$, the invariants are the magnitudes of $\frac{p}{q^{*}}$ evaluated at the poles of $Z$. The main result states that these invariants and the Hankel singular values are related by means of multiplicative majorization relations.

The paper is organized as follows. After some preliminary results on the Cauchy matrix, the issue of decay rate of the eigenvalues of one grammian is investigated in section 3 . In section 4 the general problem of the decay rate of the eigenvalues of the product of two grammians is addressed. Section 5 presents some numerical experiments. Finally, we offer some concluding remarks.

\section{Cauchy Matrices}

Our results will depend heavily on properties of Cauchy matrices. They appear fundamentally in direct formulas for solutions of Lyapunov equations. Moreover, there are closed form expressions for elements of Cholesky factors and inverses of Cauchy matrices that lend themselves to derivation of the decay estimates we seek.

Given two vectors $x, y \in \mathbb{C}^{n}$, let $\xi_{i}, \eta_{i}$ denote their $\mathrm{i}^{\text {th }}$ entry, respectively. The Cauchy matrix $\mathcal{C}(x, y) \in \mathbb{C}^{n \times n}$ is defined as follows

$$
\mathcal{C}(x, y)_{i j}=\frac{1}{\xi_{i}+\eta_{j}^{*}}, i, j=1, \cdots, n
$$

It readily follows that $\mathcal{C}(x, y)^{*}=\mathcal{C}(y, x)$, where the superscript $*$ denotes complex conjugation followed by transposition. Define the vector $d(x, y) \in \mathbb{C}^{n}$ as follows

$$
d(x, y)_{i}=\frac{\Pi_{k \neq i}\left(\xi_{i}-\xi_{k}\right)}{\Pi_{k}\left(\xi_{i}+\eta_{k}^{*}\right)} \text { and } D(x, y)=\operatorname{diag}(d(x, y))
$$

where $D(y, x)$ is the corresponding diagonal matrix. If the components of $x$ and $y$ are such that no entry of $d(x, y)$ takes a value of 0 or $\infty$, then $C(x, y)$ is nonsingular and the following result holds. 
Lemma 2.1 With the notation established above,

$$
\mathcal{C}(x, y)\left[D\left(y^{*}, x^{*}\right)\right]^{-1} \mathcal{C}\left(y^{*}, x^{*}\right)[D(x, y)]^{-1}=I_{n}
$$

The proof of this result follows from the closed form expression of the inverse of the Cauchy matrix [11]. Actually this result is quoted for real $x$ and $y$, and a slight extension is necessary to obtain the correct formula for complex $x$ and $y$.

The special case $x=y=\ell:=\left(\lambda_{1}, \lambda_{2}, \ldots, \lambda_{n}\right)^{T}$ is of interest here. It turns out that when $\mathcal{C}$ is positive definite, there are explicit formulas, due to Gohberg and Koltracht [8], for the elements of the Cholesky factors.

Lemma 2.2 If $\operatorname{Real}(\ell)<0$ then $-\mathcal{C}(\ell, \ell)$ is positive definite. Moreover, if $-\mathcal{C}(\ell, \ell)=L \Delta L^{*}$ is the Cholesky factorization, then

$$
\delta_{k}=\frac{-1}{2 \operatorname{Real}\left(\lambda_{k}\right)} \prod_{j=1}^{k-1}\left|\frac{\lambda_{k}-\lambda_{j}}{\lambda_{k}^{*}+\lambda_{j}}\right|^{2},
$$

where $\Delta=\operatorname{diag}\left(\delta_{1}, \delta_{2}, \ldots, \delta_{n}\right)$.

Remark 2.1 It is well known (see e.g. [11]) that Cauchy and the closely related Hilbert matrices are badly conditioned. Relationship (5) can be used to obtain the best diagonal conditioning of Cauchy and Hilbert matrices. For details on the solution of this problem see [1].

\section{$3 \quad$ Eigenvalue decay rates for system grammians}

\subsection{A Canonical grammian and the Cauchy kernel}

In this section, we establish the main result on the decay rates of the eigenvalues of a single Lyapunov equation. We consider the SISO case, so $B$ is a column vector which will be denoted by $b$, and the Lyapunov equation is

$$
A P+P A^{T}+b b^{T}=0 .
$$

We assume that $A$ is a stable matrix (all eigenvalues in the open left half plane). Let $A_{c}$ be the companion matrix for $A$,

$$
A_{c}=J-g e_{n}^{T}
$$

where $J$ is a left shift matrix with ones on the first subdiagonal and zeros elsewhere. The vector $g^{T}=\left(\alpha_{0}, \alpha_{1}, \ldots, \alpha_{n-1}\right)$, defines the characteristic polynomial of $A$ with

$$
q(s):=\operatorname{det}(s I-A)=s^{n}+\alpha_{n-1} s^{n-1}+\cdots+\alpha_{1} s+\alpha_{0}
$$

Define $G$ to be the solution of the canonical Lyapunov equation

$$
A_{c} G+G A_{c}^{T}+e_{1} e_{1}^{T}=0 .
$$

Let $b$ be any vector such that $(A, b)$ is controllable and let $K=\left[b, A b, A^{2} b, \ldots, A^{n-1} b\right]$ be the Krylov or controllability matrix. Since $(A, b)$ is controllable, $K$ is nonsingular and $K e_{1}=b$. It follows easily from the Cayley Hamilton theorem that, $A K=K A_{c}$. We immediately have the following lemma. 
Lemma 3.1 $P$ solves $A P+P A^{T}+b b^{T}=0$ if, and only if, $P=K G K^{T}$.

Proof: This is easily seen by multiplying on the left and right by $K$ and $K^{T}$ to get

$$
\begin{aligned}
0 & =K\left(A_{c} G+G A_{c}^{T}+e_{1} e_{1}^{T}\right) K^{T} \\
& =A K G K^{T}+K G K^{T} A^{T}+b b^{T}
\end{aligned}
$$

Since $A$ is stable, this solution is unique and the lemma is proved.

This result provides a direct relationship with the $K r y l o v$ or controllability subspace $\mathcal{K}(A, b)=\operatorname{span} K$, but further analysis is needed to derive decay rates. These rates will be a function of the eigenvalues $\lambda_{j}$ of the matrix $A$. When $A$ is diagonalizable, one has

$$
Y A_{c}=\Lambda Y
$$

where $Y$ is the Vandermonde matrix of eigenvalue powers. The $j$-th row of $Y$ is

$$
e_{j}^{T} Y=\left[1, \lambda_{j}, \lambda_{j}^{2}, \ldots, \lambda_{j}^{n-1}\right],
$$

and $\Lambda=\operatorname{diag}\left(\lambda_{1}, \lambda_{2}, \ldots, \lambda_{n}\right)$. We shall define the Cauchy kernel to be the matrix

$$
\mathcal{C}:=Y G Y^{*}
$$

This kernel will provide the decay rates we seek due to the following result.

Lemma 3.2 Let $X$ be a matrix of right eigenvectors for $A$ so that $A X=X \Lambda$ and we assume the columns of $X$ each have unit norm. Then

$$
\mathcal{C}_{i j}=\frac{-1}{\lambda_{i}+\lambda_{j}^{*}}
$$

is Hermitian positive definite and

$$
P=X_{b} \mathcal{C} X_{b}^{*}
$$

where $X_{b}=X \operatorname{diag}(\hat{b})$ with $\hat{b}=X^{-1} b$.

Proof : First observe that

$$
\begin{aligned}
K & =\left[b, A b, A^{2} b, \ldots, A^{n-1} b\right] \\
& =\left[X \hat{b}, X \Lambda \hat{b}, X \Lambda^{2} \hat{b}, \ldots, X \Lambda^{n-1} \hat{b}\right] \\
& =X_{b} Y
\end{aligned}
$$

From Lemma 3.1 we have $P=K G K^{T}=X_{b} Y G\left(X_{b} Y\right)^{*}=X_{b} \mathcal{C} X_{b}^{*}$. Since the pair $(A, b)$ is controllable, $b$ cannot be orthogonal to any left eigenvector of $A$. Hence, no component of $\hat{b}=X^{-1} b$ is zero and the matrix $\operatorname{diag}(\hat{b})$ is nonsingular. Moreover,

$$
A X_{b}=X_{b} \Lambda \text { and } X_{b}^{-1} b=e,
$$

where $e^{T}=(1,1, \ldots, 1)$. Therefore,

$$
0=X_{b}^{-1}\left(A P+P A^{T}+b b^{T}\right) X_{b}^{-*}=\Lambda \mathcal{C}+\mathcal{C} \Lambda^{*}+e e^{T} .
$$


By inspection, one finds that $\mathcal{C}$ is a Cauchy matrix with $\mathcal{C}_{i j}=\frac{-1}{\lambda_{i}+\lambda_{j}^{*}}$. Since $A$ is stable, $\Lambda$ is stable and $\mathcal{C}$ must be positive definite. This concludes the proof.

We are now prepared to derive a decay rate based upon the eigenvalues of $A$. Since $\mathcal{C}$ is positive definite, it has a Cholesky factorization. Moreover, if diagonal pivoting is included to bring the maximum diagonal element to the pivot position at each stage of the factorization, we may assume an ordering of the eigenvalues of $A$ and hence a symmetric permutation of the rows and columns of $\mathcal{C}$ such that

$$
\mathcal{C}=L \Delta L^{*}
$$

with $\Delta=\operatorname{diag}\left(\delta_{1}, \delta_{2}, \ldots, \delta_{n}\right)$ and with $L$ unit lower triangular and such that each column $L e_{j}$ satisfies $\left\|L e_{j}\right\|_{\infty}=1$. The explicit formula in Lemma 2.2 gives $\delta_{k}=\frac{-1}{2 \operatorname{Real}\left(\lambda_{k}\right)} \prod_{j=1}^{k-1}\left|\frac{\lambda_{k}-\lambda_{j}}{\lambda_{k}^{*}+\lambda_{j}}\right|^{2}$ in terms of the eigenvalues of $A$ and one may think of the diagonal pivoting in the Cholesky factorization as a means to order (i.e. index) these eigenvalues. Let $\lambda(A)$ denote the spectrum of $A$. If the first $k-1$ eigenvalues $\mathcal{S}_{k-1} \equiv\left\{\lambda_{j}: 1 \leq j \leq k-1\right\} \subset \lambda(A)$ have been selected and indexed, then the $k$-th eigenvalue $\lambda_{k}$ is selected according to

$$
\lambda_{k}=\operatorname{argmax}\left\{\frac{-1}{2 \operatorname{Real}(\lambda)} \prod_{j=1}^{k-1}\left|\frac{\lambda-\lambda_{j}}{\lambda^{*}+\lambda_{j}}\right|^{2}: \lambda \in \lambda(A) / \mathcal{S}_{k-1}\right\} .
$$

We shall call this selection the Cholesky ordering. Given the eigenvalues with any initial ordering, the Cholesky ordering and the diagonal elements of the corresponding Cholesky factorization may be computed with the following algorithm.

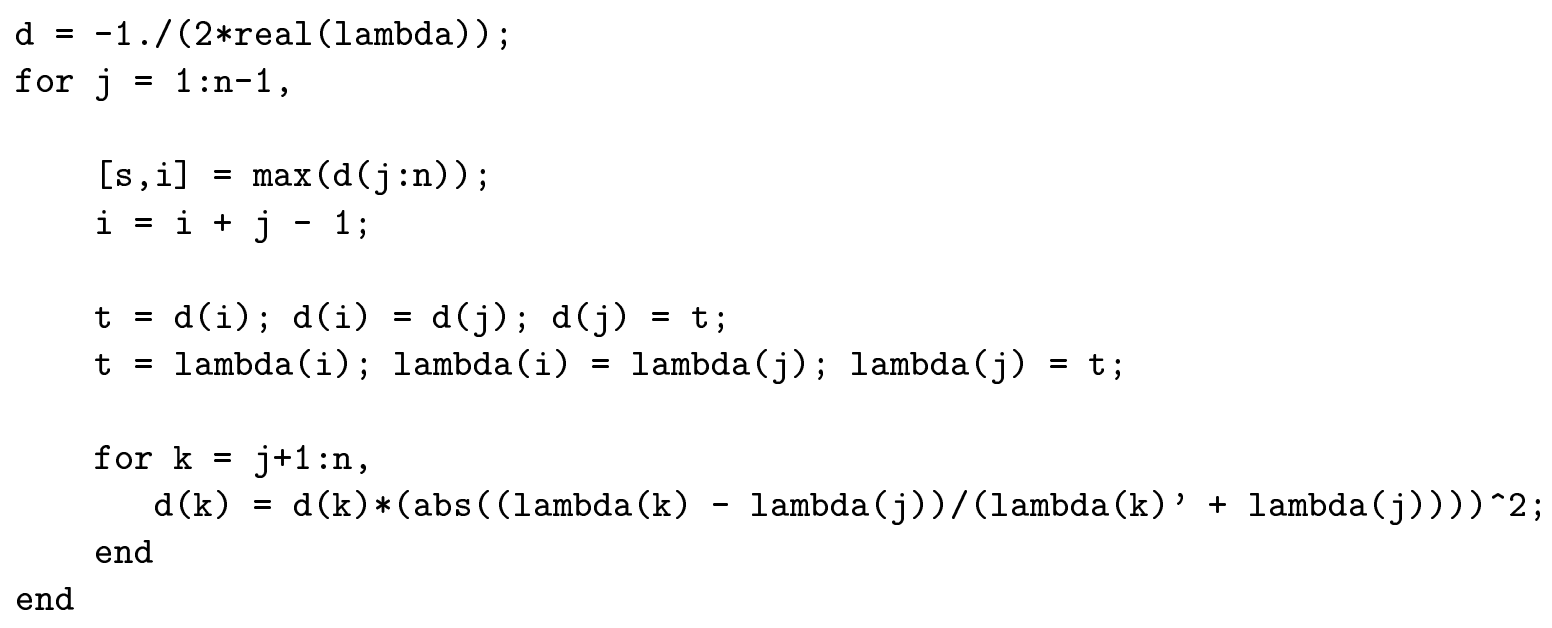

Now, given a fixed $\lambda$ in the open left halfplane, the function

$$
\phi(\zeta)=\frac{\lambda-\zeta}{\lambda^{*}+\zeta}
$$

is a linear fractional transformation that maps the open left halfplane onto the open unit disk. Thus, $\left|\phi\left(\lambda_{j}\right)\right|<1$ for every $\lambda_{j} \in \lambda(A)$. From this, we may conclude that the sequence $\left\{\delta_{j}\right\}$ is decreasing with $\delta_{1}=\frac{-1}{2 \min \left\{\text { Real }\left(\lambda_{i}\right)\right\}}=\max \left|\mathcal{C}_{i j}\right|$. These results may be summarized in the following theorem. 
Theorem 3.1 Let $P$ solve the Lyapunov equation (6). Let $P_{k}=\sum_{j=1}^{k} \delta_{j} z_{j} z_{j}^{*}$, with $\delta_{1} \geq \delta_{2} \geq \ldots \geq$ $\delta_{n}>0$ given above and where $z_{j}=X_{b} L e_{j}$. Then

$$
\left\|P-P_{k}\right\|_{\infty} \leq n(n-k) \delta_{k+1}\left(\kappa_{\infty}(X)\|b\|_{\infty}\right)^{2} .
$$

Proof: The previous discussion has established

$$
P=\sum_{j=1}^{n} \delta_{j} z_{j} z_{j}^{*} .
$$

Now, since $\left\|L e_{j}\right\|_{\infty}=1$, we have

$$
\begin{aligned}
\left\|z_{j}\right\|_{\infty} & \leq\|X\|_{\infty}\|\hat{b}\|_{\infty}\left\|L e_{j}\right\|_{\infty} \\
& =\|X\|_{\infty}\left\|X^{-1} b\right\|_{\infty} \\
& \leq \kappa_{\infty}(X)\|b\|_{\infty}
\end{aligned}
$$

The final result follows from this estimate together with $\left\|z_{j} z_{j}^{*}\right\|_{\infty}=\left\|z_{j}\right\|_{\infty}\left\|z_{j}^{*}\right\|_{1} \leq n\left\|z_{j}\right\|_{\infty}^{2}$.

Therefore, when $A$ has an eigenvector basis that is well conditioned, the norms of the vectors $z_{j}$ are uniformly bounded by a modest constant and hence the ratio $\delta_{k+1} / \delta_{1}$ gives an order of magnitude relative error estimate for a rank $k$ approximation to $P$. Departure from normality will increase the condition number of $X$ and render this bound useless. One might see the low rank phenomenon accentuated through tiny components of $\hat{b}$. On the other hand, the components of $\hat{b}$ may be magnified in a way that cancels the effects of the rapidly decaying $\delta_{j}$.

\subsection{Generalization to MIMO}

The bounds of the previous section only apply to the case where $B=b$ is a single vector. However, this result still has implications for the general case. Let $B=\left[b_{1}, b_{2}, \ldots, b_{m}\right]$, where $b_{i} \in \mathbb{R}^{n}, i=1,2, \ldots, m$. Note that Equation (2) may be written as

$$
A P+P A^{T}+\sum_{i=1}^{m} b_{i} b_{i}^{T}=0
$$

Let $P_{i}$ be the solution to

$$
A P_{i}+P_{i} A^{T}+b_{i} b_{i}^{T}=0, \quad i=1,2, \cdots, m .
$$

From (3.1) above, we have

$$
P_{i}=X_{i} \mathcal{C} X_{i}^{*}=X_{i} L D L^{*} X_{i}^{*} \text { where } X_{i}=X \operatorname{diag}\left(X^{-1} b_{i}\right) .
$$

Let

$$
Z_{j} \equiv\left[X_{1} L e_{j}, X_{2} L e_{j}, \ldots, X_{m} L e_{j}\right]=\left[z_{1 j}, z_{2 j}, \ldots, z_{m j}\right],
$$

where $z_{i j}=X \operatorname{diag}\left(X^{-1} b_{i}\right) L e_{j}$. Linearity of the Lyapunov equation yields

$$
P=\sum_{i=1}^{m} P_{i}=\sum_{j=1}^{n} \delta_{j} Z_{j} Z_{j}^{*} .
$$

With this notation we may establish the following result. 
Theorem 3.2 Let $\hat{P}_{k m}=\sum_{j=1}^{k} \delta_{j} Z_{j} Z_{j}^{*}$. If $\frac{\delta_{k}}{\delta_{1}}<\epsilon$, then $\hat{P}_{k m}$ is an approximation to $P$ of rank at most km which satisfies

$$
\left\|P-\hat{P}_{k m}\right\|_{\infty} \leq \epsilon \delta_{1}(m n)(n-k)\left(\kappa_{\infty}(X)\|B\|_{1}\right)^{2},
$$

with $\delta_{1} \approx\|P\|_{2}$.

Proof: Since $Z_{j} Z_{j}^{*}=\sum_{i=1}^{m} z_{i j} z_{i j}^{*}$, it follows that

$$
\begin{aligned}
\left\|Z_{j} Z_{j}^{*}\right\|_{\infty} & \leq \sum_{i=1}^{m}\left\|z_{i j} z_{i j}^{*}\right\|_{\infty} \\
& \leq m \max _{i}\left\|z_{i j} z_{i j}^{*}\right\|_{\infty} \\
& \leq m \max _{i} n\left(\kappa_{\infty}(X)\left\|b_{i}\right\|_{\infty}\right)^{2} \\
& \leq m n\left(\kappa_{\infty}(X)\|B\|_{1}\right)^{2},
\end{aligned}
$$

where the estimates of Theorem 3.1 are applied to each $\left\|z_{i j} z_{i j}^{*}\right\|_{\infty}$.

Obviously, there is a close connection between these bounds and the behavior of the Krylov (controllability) sequence $B, A B, A^{2} B, \ldots, A^{n-1} B$. The following well known result makes this connection precise.

Proposition 3.1 Let $A \in \mathbb{R}^{n \times n}$ be stable and $B \in \mathbb{R}^{n \times m}$. Suppose $P$ is the unique solution to $A P+$ $P A^{T}+B B^{T}=0$. and let $K=\left[B, A B, A^{2} B, \ldots, A^{n-1} B\right]$. Then $\operatorname{Rank}(P)=\operatorname{Rank}(K)$.

A proof of this result may be found, for instance, in [2]. This fact is numerically reflected in our decay rates.

\section{Decay rate of the Hankel singular values}

The estimates of the previous section are useful but fairly crude. In this section we present a set of bounds based on a new set of system invariants. The theory requires us to consider the transfer function of the system: $Z=\frac{p}{q}$. The invariants we introduce are the magnitudes of $\frac{p}{q^{*}}$ evaluated at the poles of $Z$. The main result states that these invariants and the Hankel singular values are related by means of multiplicative majorization relations.

Once again, we consider the SISO case, and we will assume for convenience that the eigenvalues $\lambda_{i}$ of $A$ are distinct and that the system is stable. The usual assumption of minimality (controllability and observability) will also be made.

The Hankel singular values of the system $\boldsymbol{\Sigma}$ are defined as the square roots of the eigenvalues of the product of the controllability grammian $P$ and the observability grammian $Q$ defined by (1):

$$
\sigma_{i}(\boldsymbol{\Sigma})=\sqrt{\lambda_{i}(P Q)}, i=1, \cdots, n
$$

These quantities are assumed ordered in deceasing order $\sigma_{i} \geq \sigma_{i+1}$. It is well known that these Hankel singular values are invariant under state space transformations. Furthermore, they turn out to be the singular values of the so-called Hankel operator associated with the system. For details we refer to [2]. 
We now introduce a new set of system invariants as follows. Let the transfer function be

$$
Z(s)=C(s I-A)^{-1} B+D_{0}=\frac{p(s)}{q(s)}
$$

Due to the minimality of the above realization, the eigenvalues of $A$ are the same as the poles of $Z(s)$, that is, the roots of the denominator polynomial $q(s)$. Let $q(s)$ be as defined in (7). We will make use of the standard definition

$$
q(s)^{*}=q^{*}(-s)=\sum_{k=0}^{n} \alpha_{k}^{*}(-s)^{k}
$$

We now define the following quantities

$$
\gamma_{i}=\frac{p\left(\lambda_{i}\right)}{q^{*}\left(\lambda_{i}\right)}=\left.\frac{p(s)}{q(s)^{*}}\right|_{s=\lambda_{i}},\left|\gamma_{i}\right| \geq\left|\gamma_{i+1}\right|, i=1, \cdots, n-1
$$

Recall that the Hankel singular values of all-pass systems satisfy the property: $\sigma_{1}=\cdots=\sigma_{n}$. A consequence of the above definition is that the same holds for the $\gamma$ 's.

Lemma 4.1 A system is all-pass (unitary) if, and only if, the $\gamma_{i}$ are all equal: $\gamma_{1}=\cdots=\gamma_{n}$.

\subsection{Multiplicative majorization}

To state the main result of this section, we need the notion of multiplicative majorization. This is a partial ordering relation between vectors. Let $\gamma, \sigma \in \mathbb{R}^{n}$ be the vectors whose $\mathrm{i}^{\text {th }}$ entry is equal to $\gamma_{i}, \sigma_{i}$, respectively. We say $\sigma$ majorizes $\gamma$ multiplicatively, and write $|\gamma| \prec_{\mu} \sigma$, if the following relations hold:

$$
|\gamma| \prec_{\mu} \sigma \Leftrightarrow \Pi_{i=1}^{k}\left|\gamma_{i}\right| \leq \Pi_{i=1}^{k} \sigma_{i}, i=1, \cdots, n-1 \text { and } \Pi_{i=1}^{n}\left|\gamma_{i}\right|=\Pi_{i=1}^{n} \sigma_{i}
$$

The result quoted next is due to Thompson [20].

Lemma 4.2 Let the matrices $\Gamma, U, P$ satisfy the relationship $\Gamma=U P$, where $P>0$ is positive definite and $U$ is unitary. If $\gamma_{i}=\lambda_{i}(\Gamma),\left|\gamma_{i}\right| \geq\left|\gamma_{i+1}\right|$ and $\sigma_{i}=\lambda_{i}(P) \geq \sigma_{i+1}, i=1, \cdots, n-1$, then multiplicative majorization holds, namely

$$
|\gamma| \prec_{\mu} \sigma .
$$

The converse is also true, namely, given $P$ and $\gamma_{i}$ satisfying the above multiplicative majorization relations, there exist a matrix $\Gamma$, where $\gamma_{i}$ are its eigenvalues, and a unitary matrix $U$ such that $\Gamma=U P$. The main result of this section is

Theorem 4.1 The vector of Hankel singular values $\sigma$ majorizes the absolute value of the vector of new invariants $\gamma$ multiplicatively:

$$
|\gamma| \prec_{\mu} \sigma
$$


Remark 4.1 (a) The last relationship of the main result, namely that the product the $\left|\gamma_{i}\right|$ is equal to the product of the Hankel singular values was first reported in the discrete-time case, by Mullis and Roberts [16].

(b) It follows from the majorization inequalities that $\prod_{i=1}^{k}\left|\gamma_{n-i+1}\right| \geq \prod_{i=1}^{k} \sigma_{n-i+1}$, for $i=1,2, \cdots, n-1$, and with equality holding for $i=n$. This implies

$$
\sum_{i=1}^{k} \log \left|\gamma_{n-i+1}\right| \geq \sum_{i=1}^{k} \log \sigma_{n-i+1}
$$

that is, the logarithmic sum of the tail of the Hankel singular values can be bounded above by the logarithmic sum of the tail of the $\gamma_{i}$.

\subsection{Interpretation of Theorem 4.1}

The issue in balanced model reduction is how fast the Hankel singular values decay to zero. In many cases the poles (natural frequencies) of the system are known together with a state space realization $\boldsymbol{\Sigma}=\left(\begin{array}{c|c}A & B \\ \hline C & D_{0}\end{array}\right)$. Thus in principle, one can compute the $\gamma_{i}$, with relatively small computational effort. The main theorem then says that the (discrete) curve whose $\mathrm{k}^{\text {th }}$ value is the product $\Pi_{i=1}^{k}\left|\gamma_{i}\right|$, is of importance. It is actually best to plot the logarithm of this curve, namely $\sum_{i=1}^{k} \log \left|\gamma_{i}\right|$, because the $\gamma_{i}$ tend to decrease rapidly and their product even more so. The main result asserts that given this curve, the corresponding curve for the Hankel singular values $\sum_{i=1}^{k} \log \sigma_{i}$, will remain above, and in addition the two curves will have to converge at the last point $\sum_{i=1}^{n} \log \sigma_{i}=\sum_{i=1}^{n} \log \left|\gamma_{i}\right|$. Furthermore the curves are monotone decreasing.

Stated differently, let $\sigma_{k}$ be known. Then the $\sigma_{\ell}, \ell>k$, will lie in the region bounded from above by $\log \sigma_{k}$ and $\sum \log \left|\gamma_{k}\right|$. This is depicted for a simple case, on the left-hand side of Figure 1.

Remark 4.2 A consequence of the main result is that non-minimum phase systems are harder to approximate than minimum phase ones. Recall that minimum-phase systems are systems with stable zeros, i.e. zeros which lie in the left-half of the complex plane. This follows from the definition (10) since, assuming that the poles are the same, each $\gamma_{i}$ for a minimum-phase system will be smaller than the $\gamma_{i}$ for a system with all zeros in the right-half of the complex plane.

\subsection{The proof of theorem 4.1}

Consider again the system $\boldsymbol{\Sigma}$ where the previous assumptions hold. Due to the fact that the poles are distinct, the transfer function can be decomposed in a partial fraction expansion of the type:

$$
Z(s)=\frac{p(s)}{q(s)}=\frac{p(s)}{\Pi_{i=1}^{n}\left(s-\lambda_{i}\right)}=D_{0}+\sum_{i=1}^{n} \frac{b_{i}}{s-\lambda_{i}}
$$

It follows that $p(s)=D_{0} q(s)+\sum_{i=1}^{n} b_{i} \Pi_{j \neq i}\left(s-\lambda_{j}\right)$. Therefore $p\left(\lambda_{i}\right)=b_{i} \Pi_{j \neq i}\left(\lambda_{i}-\lambda_{j}\right)$. Notice also that

$$
\left.q(s)^{*}\right|_{s=\lambda_{i}}=\Pi_{j=1}^{n}\left(\lambda_{i}-\lambda_{j}^{*}\right)
$$



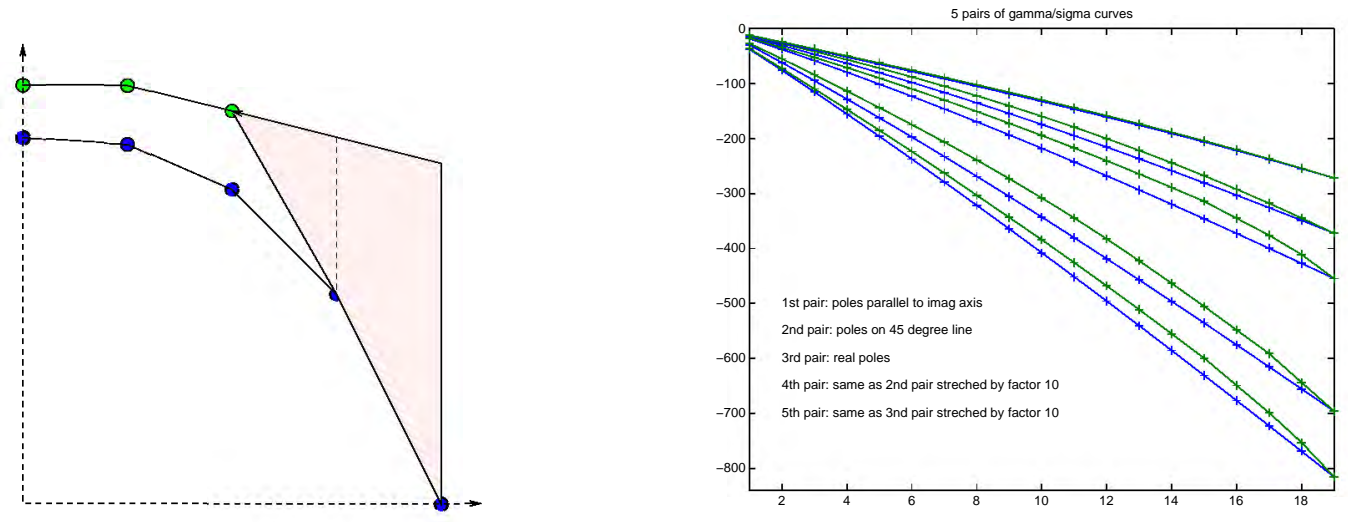

Figure 1: 5 pairs of $\gamma / \sigma$ curves for all-pole systems of order 19 are depicted.The $\gamma$ curve is always the lower one. The units on the y-axis are orders of magnitude. The curves of the right-hand side figure are from upper to lower, as follows. The first system has poles with real part equal to -1 . The next has poles which are on a 45 degree angle with respect to the negative real axis. The third one has real poles only. Finally the last two pairs have their poles spread apart by a factor of 10 with respect to the previous two pairs.

From the partial fraction decomposition follows the state space realization

$$
\boldsymbol{\Sigma}=\left(\begin{array}{c|c}
A & B \\
\hline C & D_{0}
\end{array}\right), A=\operatorname{diag}\left(\left[\lambda_{1}, \cdots, \lambda_{n}\right]\right), B=\left(\begin{array}{c}
1 \\
\vdots \\
1
\end{array}\right), C=\left[\begin{array}{lll}
b_{1} & \cdots & b_{n}
\end{array}\right]
$$

Then the controllability grammian is equal to the Cauchy matrix with $x=y=\ell$ (the vector of eigenvalues $\lambda_{j}$ ), while the observability grammian is a diagonally scaled version of the Cauchy matrix with $x=y=\bar{\ell}$ (the vector of complex conjugate eigenvalues). Let $\mathcal{B}$ be the diagonal matrix whose entries on the diagonal are $b_{i}$. Then

$$
P=-\mathcal{C}(\ell, \ell)=-\left(\begin{array}{cccc}
\frac{1}{\lambda_{1}+\lambda_{1}^{*}} & \frac{1}{\lambda_{1}+\lambda_{2}^{*}} & \cdots & \frac{1}{\lambda_{1}+\lambda_{n}^{*}} \\
\frac{1}{\lambda_{2}+\lambda_{1}^{*}} & \frac{1}{\lambda_{2}+\lambda_{2}^{*}} & \cdots & \frac{1}{\lambda_{2}+\lambda_{n}^{*}} \\
\vdots & \vdots & \ddots & \vdots \\
\frac{1}{\lambda_{n}+\lambda_{1}^{*}} & \frac{1}{\lambda_{n}+\lambda_{2}^{*}} & \cdots & \frac{1}{\lambda_{n}+\lambda_{n}^{*}}
\end{array}\right) \in \mathbb{C}^{n \times n} \Rightarrow Q=-\mathcal{B C}\left(\ell^{*}, \ell^{*}\right) \mathcal{B}^{*}
$$

From the above remarks it follows that $d(\ell, \ell)_{i}=\frac{p\left(\lambda_{i}\right)}{q^{*}\left(\lambda_{i}\right)}=b_{i} \frac{\Pi_{j \neq i}\left(\lambda_{i}-\lambda_{j}\right)}{\Pi_{j}\left(\lambda_{i}+\lambda_{j}^{*}\right)}$. Thus from the previous discussion and in particular Lemma 2.1, we conclude that

$$
\mathcal{C}(\ell, \ell) D\left(\ell^{*}, \ell^{*}\right)^{-1} \mathcal{C}\left(\ell^{*}, \ell^{*}\right) D(\ell, \ell)^{-1}=I_{n}
$$

This implies

$$
P\left[\mathcal{B D}\left(\ell^{*}, \ell^{*}\right)\right]^{-1} Q\left[D(\ell, \ell) \mathcal{B}^{*}\right]^{-1}=I_{n}
$$


From here we proceed as follows. Let $T$ be a balancing transformation, i.e. $T P T^{*}=T^{-*} Q T^{-1}=\Sigma$, where $\Sigma=\operatorname{diag}\left(\sigma_{1}, \cdots, \sigma_{n}\right)$, are the Hankel singular values of $\boldsymbol{\Sigma}$. Then

$$
\begin{aligned}
& \underbrace{T P T^{*}}_{\Sigma} \underbrace{T^{-*}\left(\mathcal{B} D\left(\ell^{*}, \ell^{*}\right)\right)^{-1} T^{*}}_{\hat{D}^{-1}} \underbrace{T^{-*} Q T^{-1}}_{\Sigma} \underbrace{T\left(D(\ell, \ell) \mathcal{B}^{*}\right)^{-1} T^{-1}}_{\hat{D}^{-*}}=\Sigma \hat{D}^{-*} \Sigma \hat{D}^{-1}=I_{n} \\
\Rightarrow & \underbrace{\sqrt{\Sigma} \hat{D}^{-*} \sqrt{\Sigma}}_{U^{*}} \underbrace{\sqrt{\Sigma} \hat{D}^{-1} \sqrt{\Sigma}}_{U}=I_{n}
\end{aligned}
$$

where $\sqrt{\Sigma}$ denote the square root of $\Sigma$, i.e. $\sqrt{\Sigma} \sqrt{\Sigma}=\Sigma$; then the above equality implies $\sqrt{\Sigma} \hat{D}^{-1} \sqrt{\Sigma}=U$, where $U$ is unitary $U U^{*}=U^{*} U=I_{n}$. Therefore, $\sqrt{\Sigma} U^{*} \sqrt{\Sigma}=\hat{D}$. Then, since, $\lambda_{i}(\hat{D})=d(\ell, \ell)_{i}$, and since $\lambda_{i}\left(\sqrt{\Sigma} U^{*} \sqrt{\Sigma}\right)=\lambda_{i}(U \Sigma)$, Lemma 4.2 together with (10), yield the desired result (12).

\subsection{A sharpening of the main result}

Recall (3) and (4). We associate with the $\xi_{i}$ 's and $\eta_{i}$ 's the following vectors $\delta^{\uparrow} \in \mathbb{R}^{n}$ and $\delta^{\downarrow} \in \mathbb{R}^{n}$ :

$$
\delta_{k}^{\uparrow}=\frac{\left|b_{k}\right|}{\xi_{k}+\eta_{k}^{*}} \Pi_{i=1}^{k-1}\left|\frac{\xi_{k}-\xi_{i}}{\xi_{k}+\eta_{i}^{*}}\right|^{2}, \quad \delta_{k}^{\downarrow}=\frac{\left|b_{k}\right|}{\xi_{k}+\eta_{k}^{*}} \Pi_{i=k+1}^{n}\left|\frac{\xi_{k}-\xi_{i}}{\xi_{k}+\eta_{i}^{*}}\right|^{2}, k=1,2, \cdots, n
$$

It will be assumed in the sequel that the $\xi_{i}$ are arranged so that the entries of $\delta^{\uparrow}$ turn out to be in decreasing Cauchy ordering: $\delta_{k}^{\uparrow} \geq \delta_{k+1}^{\uparrow}$. From our earlier discussion it follows that if the system is given by (13), the $\gamma_{i}$ are:

$$
\gamma_{k}=\frac{b_{k}}{\xi_{k}+\eta_{k}^{*}} \Pi_{i \neq k} \frac{\xi_{k}-\xi_{i}}{\xi_{k}+\eta_{i}^{*}}
$$

Notice that $\delta^{\uparrow} \odot \delta^{\downarrow}=\gamma \odot \bar{\gamma}=|\gamma|^{2}$, where $\odot$ denotes point-wise multiplication. It should also be noticed that as mentioned earlier [8], the L-D-U factorization of the Cauchy matrix can be written explicitly as $\mathcal{C}=L \Delta U$, where $L$ is lower triangular with ones on the diagonal, $U$ is upper triangular with ones on the diagonal, and $\Delta=\operatorname{diag} \delta^{\uparrow}$.

Suppose that we are given a matrix $A=\operatorname{diag}\left(\lambda_{1}, \cdots, \lambda_{n}\right) \in \mathbb{R}^{n \times n}$, and $B=\left[\begin{array}{llll}b_{1} & b_{2} & \cdots & b_{n}\end{array}\right]^{T} \in \mathbb{C}^{n}$. In this case the solution of the Lyapunov equation $A P+P A^{*}+B B^{*}=0$ is precisely the Cauchy matrix scaled by $B: \mathcal{C}_{i, j}=\frac{b_{i} b_{j}^{*}}{\lambda_{i}+\lambda_{j}^{*}}$. Because of the above mentioned fact on the L-D-U factorization we have the following result due to Alfred Horn [13]; it states that the vector of eigenvalues of a positive definite matrix majorizes multiplicatively the vector whose $\mathrm{i}$-th entry is the quotient of the determinants of the i-th over the (i-1)-st principal minors of the matrix.

Lemma 4.3 Let $P$ be the solution to the Lyapunov equation with eigenvalues $\lambda_{i}(P)$. Then, the vector $\delta^{\uparrow}$, is majorized multiplicatively by the vector of eigenvalues $\lambda(P)$. Furthermore the former multiplicatively majorizes the vector $\gamma$ :

$$
|\gamma| \prec_{\mu} \delta^{\uparrow} \prec_{\mu} \lambda(P)
$$

Remark 4.3 (a) In [1] it was shown that the optimal conditioning of Cauchy matrices by means of diagonal scaling is given by

$$
\beta_{k}=\left|\xi_{k}+\eta_{k}^{*}\right| \prod_{i \neq k}\left|\frac{\xi_{k}+\eta_{i}^{*}}{\xi_{k}-\xi_{i}}\right|
$$


This implies that for optimally conditioned Lyapunov equations we must have $\delta^{\uparrow} \odot \delta^{\downarrow}=e^{T}=\left[\begin{array}{llll}1 & 1 & \cdots & 1\end{array}\right]$. (b) The inequalities (16) are a refined version of the result concerning the decay rate of the eigenvalues of a single grammian; see theorem 3.1.

\subsection{Connection with Fuhrmann's results}

In reference [6], the signed Hankel singular values (i.e. the eigenvalues) of the Hankel operator $\mu$, and the corresponding eigenvectors, are characterized by means of a polynomial equation as follows:

$$
p r=\mu q^{*} r^{*}+q \pi
$$

where $Z=\frac{p}{q}$ is the transfer function of the system, $\sigma$ is a Hankel singular value, $\mu=\epsilon \sigma, \epsilon= \pm 1$, is the corresponding signed singular value, $(\cdot)^{*}$ is as defined earlier, and $r, \pi$ are polynomials to be determined. Let $a, b$ be polynomials satisfying the Bezout equation $a q+b q^{*}=1$. Then the coefficients of the polynomial $r$ and the eigenvalues $\mu$ of the Hankel operator are determined from the eigenvalue decomposition of the matrix

$$
M=K b(A) p(A)
$$

where $A$ is any matrix with characteristic polynomial equal to $q$ and $K=\operatorname{diag}(1,-1,1,-1, \cdots)$. For details of this polynomial approach see $[6]$.

We notice that (17) can be rewritten as follows:

$$
\frac{p}{q^{*}}=\mu \frac{r^{*}}{r}+\frac{q}{q^{*}} \frac{\pi}{r} \Rightarrow \gamma_{i}=\left.\frac{p}{q^{*}}\right|_{s=\lambda_{i}}=\left.\mu \frac{r^{*}}{r}\right|_{s=\lambda_{i}}
$$

where $-\lambda_{i}$ are the roots of $q$ (poles of $\boldsymbol{\Sigma}$ ). It follows therefore that $\gamma_{i}$ is equal to some singular value times the magnitude of the corresponding all-pass $\frac{r^{*}}{r}$, evaluated at $s=\lambda_{i}$; to stress the dependency on the $\mathrm{j}^{\text {th }}$ singular value we write $r_{j}$ instead of $r$ :

$$
\gamma_{i}=\frac{p\left(\lambda_{i}\right)}{q^{*}\left(\lambda_{i}\right)}=\sigma_{j} \frac{r_{j}^{*}\left(\lambda_{i}\right)}{r_{j}\left(\lambda_{i}\right)}, \quad j=1, \cdots, n
$$

Thus the ratio of $\gamma_{i}$ and $\sigma_{j}$ is a quantity depending on the all-pass function defined by the polynomial $r$ which in turn defines the eigenvectors of the Hankel operator.

Following a presentation of Theorem 4.1, at the Conference on Approximation and Optimization which took place in Guatemala, March 26-29, 2001, Paul Fuhrmann remarked a few days later in a private communication to the first author that in [6], there is a Toeplitz operator which in retrospect can be seen to have as eigenvalues the $\gamma_{i}$ and as singular values the $\sigma_{i}$. This fact implies the multiplicative majorization relationships (12). Work is currently underway to elaborate on this observation. In particular the system theoretic interpretation of this Toeplitz operator is one of the issues.

\section{$5 \quad$ Numerical Examples and Discussion}

In this section, we illustrate the effectiveness of the Cholesky estimates for decay rates of the eigenvalues of the system grammians derived in section 3 (see theorem 3.1). We have observed essentially the same quality of results for the decay rate estimates for the Hankel singular values. As the two are intimately related, we only report on the results for the eigenvalues of one grammian here. 
Even though the results of Section 3 do not establish direct bounds on the eigenvalues of $P$ they seem to predict the behavior of these eigenvalues quite well. We illustrate this with some computational results and compare our estimates to those derived by Penzl in [17]. To our knowledge these were the only eigen-decay estimates available prior to the results given here. Penzl's results are only valid for symmetric $A$. When $m=1$ (the SISO case), these bounds are

$$
\frac{\lambda_{k+1}(P)}{\lambda_{1}(P)} \leq\left(\prod_{j=0}^{k-1} \frac{\kappa(A)^{(2 j+1) /(2 k)}-1}{\kappa(A)^{(2 j+1) /(2 k)}+1}\right)^{2} .
$$

Our estimates are derived from the diagonal elements of the Cholesky factor of $\mathcal{C}$ and do not require symmetry of $A$. They are of the form

$$
\delta_{k}=\frac{-1}{2 \operatorname{Real}\left(\lambda_{k}\right)} \prod_{j=1}^{k-1}\left|\frac{\lambda_{k}-\lambda_{j}}{\overline{\lambda_{k}+\lambda_{j}}}\right|^{2},
$$

where the $\lambda_{j}$ 's have been indexed according to the Cholesky ordering imposed by diagonal pivoting.

Even though these two results are derived in very different ways, they are closely related. In fact, Penzl derives his bounds from expressions involving the same linear fractional transformations that led to our results. However, in that case, they arose from ADI approximations to the solution of (2).

Our computations indicate that a great deal may be lost in replacing estimates involving all of the eigenvalues $\lambda_{j}$ of $A$ with a condition number. In Figure 2 we show the results of our estimates versus Penzl's in comparison to the eigenvalues of $P$ where $A$ is the standard finite difference approximation to the 1-D laplacian of order 100.

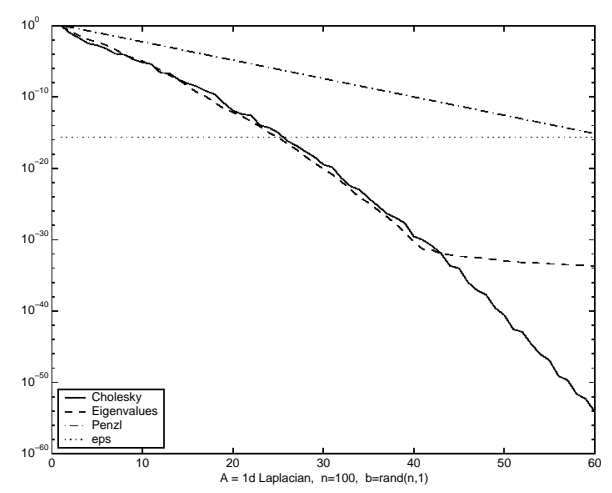

Figure 2: Comparison of Estimates: Discrete Laplacian

Figure 2 gives a semilog graph of the Cholesky estimates $\delta_{k} / \delta_{1}$, the Penzl estimates, and the actual eigenvalue ratios $\lambda_{k}(P) / \lambda_{1}(P)$ for $1 \leq k \leq 60$. The horizontal dotted line indicates where these ratios fall below machine precision eps $\approx 10^{-16}$. In Figure 3 , we show the same comparison for a random nonsymmetric $A$ of order 200 with a few eigenvalues near the imaginary axis (distance about .01).

In Figure 4 we compare Cholesky estimates to actual eigen-decay on LTI examples that are more closely related to engineering applications. Two of the examples are simple model problems, a finite element model of heat conduction in a plate with boundary controls and a finite element model of a 


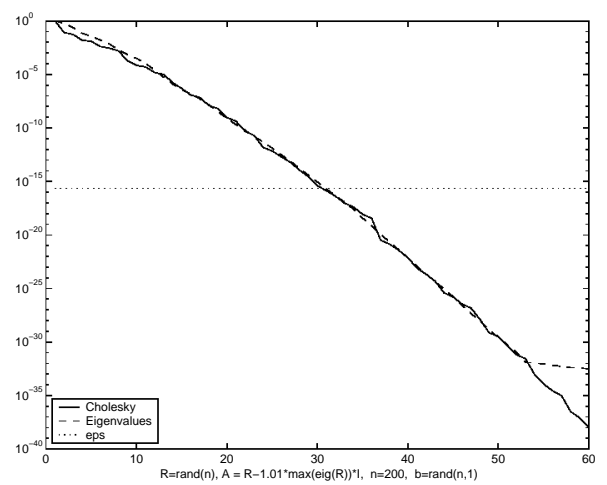

Figure 3: Comparison of Estimates: Nonsymmetric A

clamped beam with a control force applied at the free end. These are labeled "Heat model" and "Struct model" respectively. A third example labeled "CD player model" is a simplified simulation of a CD player tracking mechanism that has been described in detail in [9]. The fourth example labled "ISS 1r-c04 model" is an actual finite element discretization of the flex modes of the Zvezda Service Module of the International Space Station (ISS). There are 3 inputs, and 3 outputs, namely the roll, pitch, yaw jets, and the roll, pitch, yaw rate gyros readings, respectively. This example was provided to us by Draper Labs. Since $A$ is nonsymmetric in all of these systems, the Penzl estimates do not apply. However, the Cholesky ratios give very good approximations to the actual eigen-decay rates for all of these examples.

These results indicate that the condition number $\kappa(A)$ alone may not be enough to determine the decay rate effectively. It seems that the decay rate really depends on the full spectrum of $A$ as indicated by the Cholesky estimates. Moreover, one can easily see that clustered eigenvalues of $A$ can make the Cauchy kernel have very low numerical rank (hence $P$ will have very low numerical rank), but $\kappa(A)$ can be arbitrarily large at the same time. Hence the right hand side in (18) fails to predict the rapid eigen-decay rate. In fact, the eigen-decay rate can be extremely fast even when $\kappa(A)$ is huge. This is illustrated by the example shown in Figure 5 where $A$ has been constructed to have two clustered eigenvalues as follows:

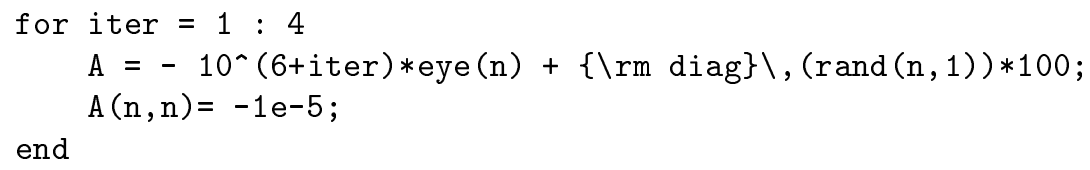

For the nonsymmetric case, Penzl [17] constructed a nice example to show that even in the SISO case, the eigen-decay rate can be arbitrarily slow. In the extreme case the ratios remain almost constant at 1. His example is: put $n=2 d+1, m=1, A=\operatorname{diag}\left(-1, A_{1}, A_{2}, \ldots, A_{d}\right) \in \mathbb{R}^{n \times n}, B=[1,1, \ldots, 1]^{T} \in \mathbb{R}^{n}$, where

$$
A_{j}=A_{j}(t)=\left[\begin{array}{cc}
-1, & j * t / d \\
-j * t / d, & -1
\end{array}\right], \quad j=1,2, \ldots, d .
$$

Penzl considered $d=50, t=10,100,1000$, from the construction of $A, \kappa(A)=\|A\|_{2}\left\|A^{-1}\right\|_{2} \simeq t$. He observed that the eigenvalue ratios are almost constant 1 for large $t$.

We tried this example with $d=300, t=10,100,10^{3}, 10^{4}$. As can be seen from Figure 6 , the eigen-decay rate of $P$ slows down when $A$ has eigenvalues with increasingly dominant imaginary parts. In [17] Penzl suggested that the dominant imaginary parts of the eigenvalues cause the decay rate to 

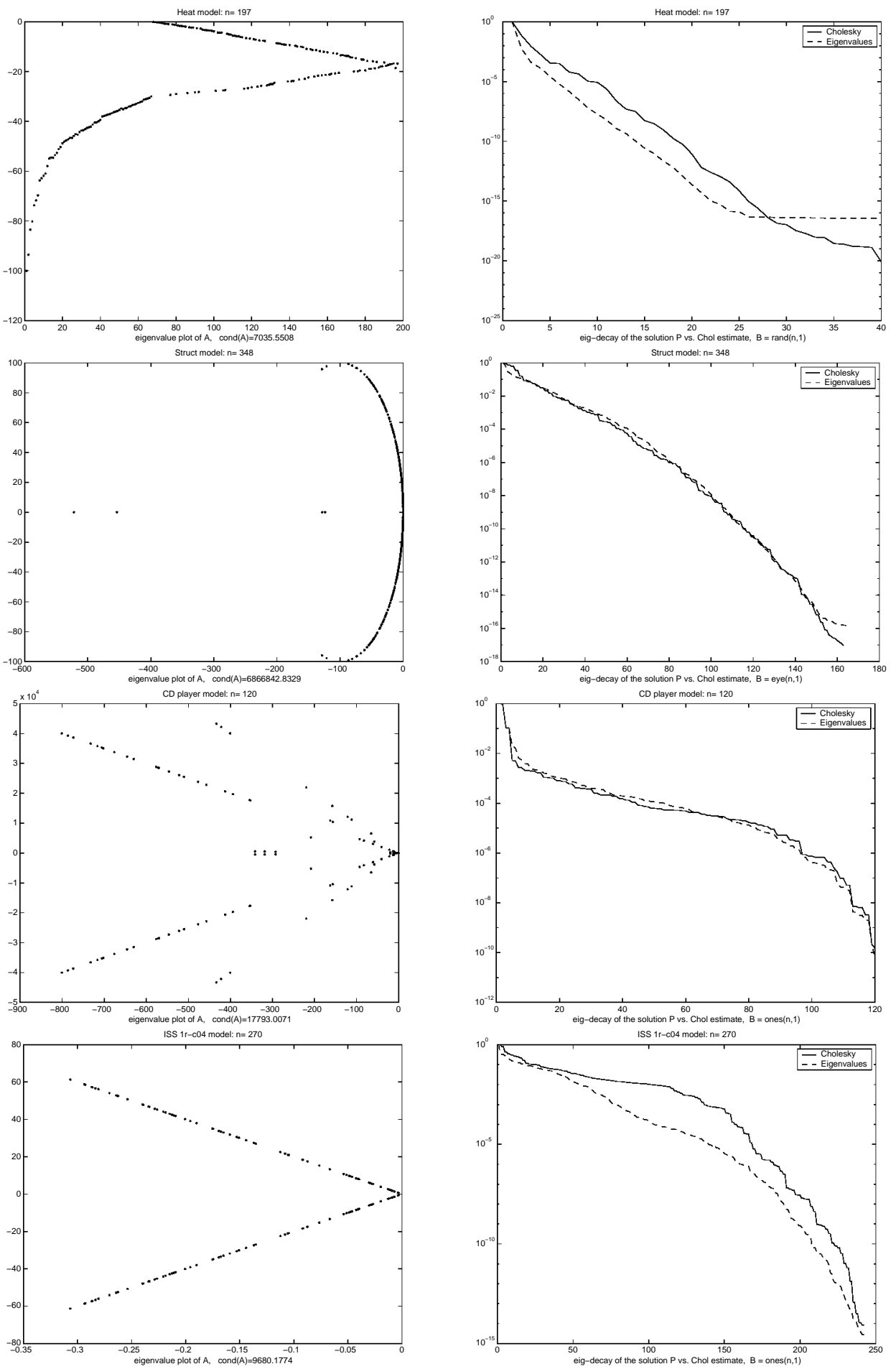

Figure 4: Eigen-decay rate vs Cholesky estimates for some real LTI systems 


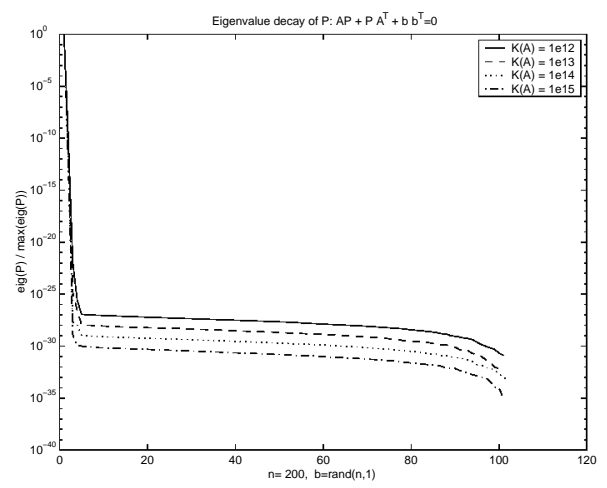

Figure 5: Fast eigen-decay rate for large cond(A)

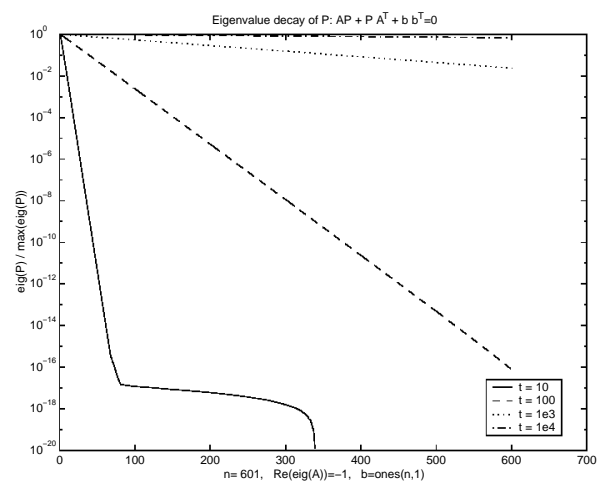

Figure 6: Decay rates for Penzl's example

slow down. This observation is relevant, but further analysis seems to indicate that relative dominance of the imaginary parts to the real parts, together with the absence of clustering in the spectrum of $A$ are very important factors.

We illustrate this in Figure 7 by constructing $A$ with eigenvalues having huge imaginary parts but with all eigenvalues clustered about 3 points: $\{-1,-1+t i,-1-t i\}$. Again we construct: $n=2 d+1$, $A=\operatorname{diag}\left(-1, A_{1}, A_{2}, \ldots, A_{d}\right) \in \mathbb{R}^{n \times n}, B=[1,1, \ldots, 1]^{T} \in \mathbb{R}^{n}$, while we modified $A_{j}$ as follows

$$
A_{j}=A_{j}(t)=\left[\begin{array}{cc}
-1, & t+j / d \\
-t-j / d, & -1
\end{array}\right], \quad j=1,2, \cdots, d .
$$

Again, we take $t=10,100,10^{3}, 10^{4}$ for comparison.

In this example, despite the presence of eigenvalues with increasingly dominant imaginary parts, the eigen-decay rate of $P$ does not deteriorate because of the clustered eigenvalues. For each $t$ in this example $A$ has only 3 clustered eigenvalues, and thus the Cauchy kernel (3) has low numerical rank for each $t$. Hence, the eigen-decay rate of $P$ continues to be fast regardless of the magnitude of $t$ as demonstrated in Figure 7. We also see that $\kappa(A)$ is irrelevant to the decay rate in this example since here $\kappa(A) \simeq t$ for each $t$. 


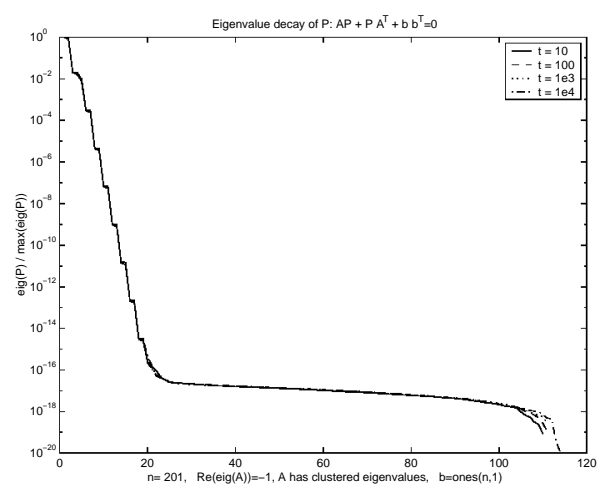

Figure 7: Fast Eigen-decay rate: $A$ has clustered eigenvalues with dominant imaginary parts

Actually (18) gives an explanation of the non-decay in Penzl's example. In this example, Real $(\lambda)$ remains constant and the increasingly dominant $\operatorname{Imag}(\lambda)$ leads to $\left|\frac{\lambda_{k}-\lambda_{j}}{\lambda_{k}+\lambda_{j}}\right| \rightarrow 1$. Hence, $\left\{\delta_{k}\right\}$ becomes nearly a constant sequence and there is very little decay of the eigenvalues.

The Cholesky estimates are not shown in Figures 5, 6, 7 to avoid the clutter of so many overlayed plots. However, in all of these cases the Cholesky ratios were computed and again they approximate the actual eigen-decay rates well. All computations were done in Matlab 5.3.0 on a Sun Spark Ultra-60 under SunOS 5.6. Machine epsilon is approximately $10^{-16}$.

\subsection{Cases of non-decay or slow-decay}

Penzl's example is one slow-decay case. In fact, the worst case is possible. Equation (2) may have the solution $P=I$ where $I$ is the identity matrix. As an example, if $T=-T^{T}$, choose $A=T-\mu e_{1} e_{1}^{T}, b=$ $\sqrt{2 \mu} e_{1}$ for some $\mu>0$. Then

$$
A I+I A^{T}+b b^{T}=0
$$

Thus, even in the SISO case, it is possible that there will be no eigen-decay for the solution of (2). We also did extensive numerical study on these non-decay cases, the most relevant reasons we found are: (i) most eigenvalues of $A$ have tiny real parts with not so tiny imaginary parts; (ii) most eigenvalues of $A$ have dominant imaginary parts and the spectrum of $A$ is not clustered relative to $\max _{j}\left|\operatorname{Real}\left(\lambda_{j}\right)\right|$. However, Figure 8 shows even in the slow-decay or non-decay case, (5) still approximates the actual decay rate well. In engineering applications, it seems to be uncommon for the conditions for slow decay to arise.

Remark 5.1 (a) The Cholesky ratio (5) only involves the eigenvalues of $A$, while $P$ depends on both $A$ and $b$, so there may be considerable discrepancy between the Cholesky ratio and the actual eigen-decay rate for some $b$. However, when $A$ has a moderately well conditioned eigenvector basis (5) usually gives an accurate prediction of the numerical rank of $P$.

(b) On the other hand, $b$ is taken into account in theorem 4.1 and in the refined bounds (16).

The result of Theorem 3.2 suggests that the rank of $P$ will increase with the rank of $B$. Figure 9 gives one example of the effect of increasing $m$ (the number of columns of $B$ ). Note in this example that 

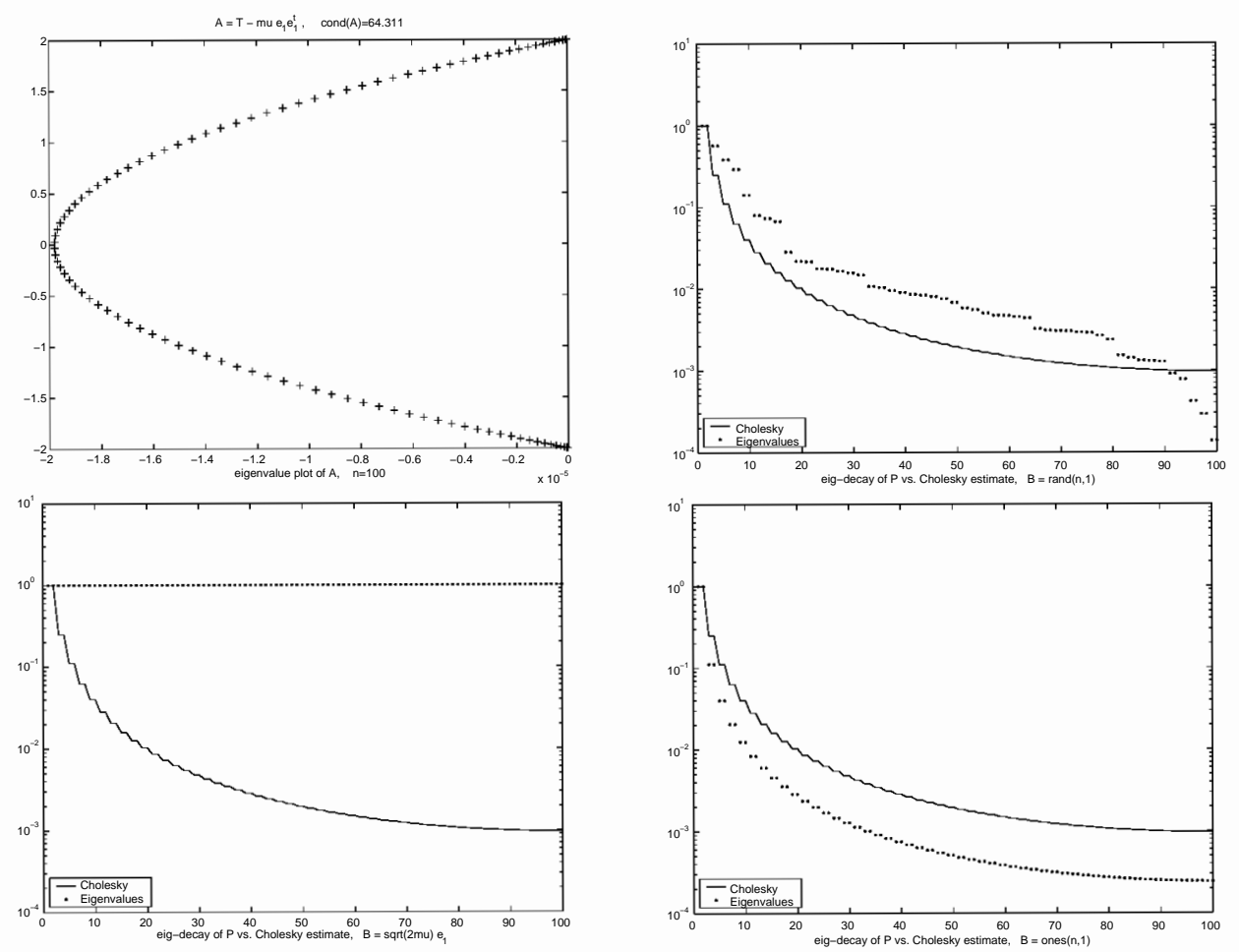

Figure 8: Eigen-decay rate and Cholesky estimates for the slow-decay cases

the spectrum of $A$ contains relatively clustered points with dominant real parts, which implies the fast eigen-decay rate.

\section{Conclusions}

In the first part of this paper we related the rank of the grammian $P$ in (2), to the diagonal elements of the diagonally pivoted Cholesky factor of the Cauchy kernel generated by the eigenvalues of $A$. We compared the resulting Cholesky estimates to others with favorable results. Our estimates seem to be quite accurate for both symmetric and nonsymmetric $A$ so long as $A$ has a moderately conditioned eigenvector basis.

In the second part we investigated the problem of decay of the Hankel singular values. We found that they satisfy multiplicative majorization inequalities with respect to a new set of invariants which we called $\gamma_{i}$.

Our results do depend upon the assumption that $A$ is diagonalizable. Further work is needed to fully understand the implications of matrices $A$ that are defective or highly non-normal. Moreover, our results depend on complete knowledge of the spectrum of $A$. Hence, they are not practical for gaining a-priori estimates for the effective rank of the grammians. It would be highly desirable to give practical and tight upper bounds on the estimates derived in the first part of the paper. 


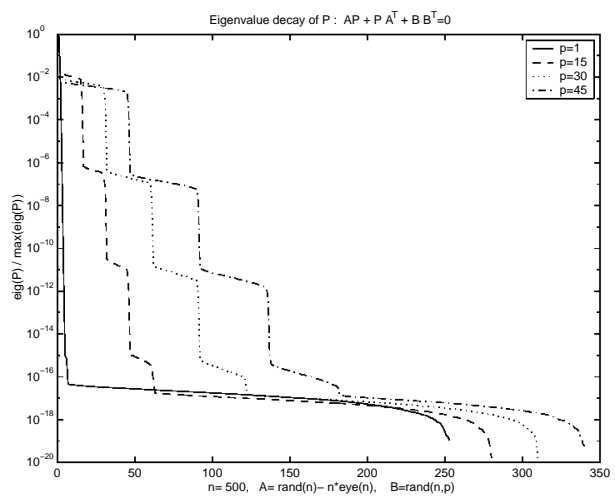

Figure 9: MIMO cases: the effect of different $p$

\section{References}

[1] A.C. Antoulas, On eigenvalues and singular values of linear dynamical systems, Proceedings of the Householder XIV Symposium, Chateau Whistler, BC, 14-18 June 1999, pages 8-11.

[2] A.C. Antoulas, Lectures on the approximation of large-scale dynamical systems, SIAM Press, to appear (2001).

[3] A.C. Antoulas, D.C. Sorensen, and S. Gugercin, A survey of model reduction methods for large-scale systems, in "Structured Matrices in Operator Theory, Numerical Analysis, Control, Signal and Image Processing," Contemporary Mathematics, edited by V. Olshevsky, AMS publications (2001).

[4] F.S.V. Bazán and Ph.L. Toint, Conditioning of infinite Hankel matrices of finite rank, Systems and Control Letters, 41: 347-359 (2000).

[5] B. Beckermann, The condition number of real Vandermonde, Krylov and positive definite Hankel matrices, Numerische Mathematik, 85: 553-577 (2000).

[6] P.A. Fuhrmann, A polynomial approach to Hankel norm and balanced approximations, Linear Algebra and its Applications, 146: 133-220 (1991).

[7] Z. Gajic, M.T.J. Qureshi, Lyapunov Matrix Equations in System Stability and Control, Academic Press, 1995

[8] I. Gohberg and I. Koltracht, Triangular factors of Cauchy and Vandermonde matrices, Integral Equations and Operator Theory, 46: 46-59 (1996).

[9] E. Grimme, D.C. Sorensen and P. Van Dooren, Model reduction of state space systems via an implicitly restarted Lanczos method, Numerical Algorithms, 12: 1-32 (1996).

[10] T. Gudmundsson, A. J. Laub, Approximate Solution of Large Sparse Lyapunov Equations, IEEE Trans on AC, AC-39: 1110-1114 (1994)

[11] N.J. Higham, Section 26.1: The Hilbert and Cauchy matrices, in "Accuracy and stability of numerical algorithms", SIAM Press, (1996).

[12] A. Hodel, K. Poolla, B. Tenison, Numerical solution of the Lyapunov equation by approximate power iteration, Linear Algebra Appl., 236: 205-230 (1996) 
[13] A. Horn, On the eigenvalues of a matrix with prescribed singular values, Proc. Amer. Math. Soc., 5: 4-7 (1954).

[14] I. Jaimoukha and E. Kasenally, Krylov Subspace methods for solving Large Lyapunov Equations, SIAM J. Numer. Anal., 31: 227-251 (1994)

[15] W. Kwon, Y. Moon, S. Ahn, Bounds in algebraic Riccati and Lyapunov equations: A survey and some new results, Inter. J. Control, 64: 377-389 (1996)

[16] C.T. Mullis and R.A. Roberts, Synthesis of minimum round-off noise fixed point digital filters, IEEE Trans. Circuits and Systems, CAS-33: 551-562 (1976).

[17] T. Penzl, Eigenvalue Decay Bounds for Solutions of Lyapunov Equations: The Symmetric Case, Systems and Control Letters, 40: 139-144 (2000).

[18] T. Penzl, A Cyclic Low-Rank Smith Method for Large Sparse Lyapunov Equations, SIAM J. Sci. Comput., 21: $1401-1418$ (2000)

[19] Y. Saad, Numerical Solution of Large Lyapunov Equations, in Signal Processing, Scattering, Operator Theory and Numerical Methods: Proceedings of the Int. Symposium MTNS-89, Vol. III, M. Kaashoek, J.H. van Schuppen, A. Ran, eds., Birkhäuser, Boston, MA, pp. 503-511 (1990)

[20] S. Sherman and C.J. Thompson, Equivalences on eigenvalues, Indiana University Mathematics Journal, 21: 807-814 (1972). 